\title{
Partial interpretations
}

FRANZ VON KUTSCHERA

\section{Statement of the problem}

Natural languages contain many expressions which are grammatically well-formed but meaningless; they are assembled from meaningful words or morphemes ${ }^{1}$ in accordance with the syntactic rules of the language but no meaning is conferred upon them by the semantic rules of the language. When we call expressions or utterances 'meaningless' here without further qualification, that will just be for the sake of brevity. We want to indicate by that term that the expressions or utterances are semantically anomalous in such a way that they will generally evoke responses like 'What do you mean?' or 'What are you talking about?' There is no implication that they are on a par with totally meaningless expressions as $\mathrm{Krz}$ is thwing.

Let us take six typical examples of such well-formed but meaningless expressions:

(1) Incompletely defined functors: Many predicates are not defined for all syntactically permissible arguments. Thus the verb to run is defined for animals with locomotive appendages, for humans, machines, fluids and for noses, not however for plants, minerals or numbers. And the German verb lachen is defined only for humans and the sun. The sentence Der Mond lacht, though constructed grammatically just as Die Sonne lacht, has, in distinction to the latter, no meaning.

(2) Non-existing objects: Sentences about objects which do not exist or no longer exist form a significant sub-category of example (I). The sentences Odysseus is (now) shaving himself and Eisenhower is (now) sick are meaningless but not the sentences Professor Snell is dreaming of Odysseus or Nixon remembers Eisenhower. Thus many predicates are defined for non-existent objects while others are not. Since the question of whether a human being is alive or dead is purely empirical, syntax cannot refer to this distinction.

(3) Invalid presuppositions: A presupposition of a statement or utterance $A$ is a state of affairs which is not itself asserted in $A$, but which

${ }^{1}$ Meaningless expressions do not rate as words of the lexicon upon which the syntax is based. 
must be the case if both $A$ and the (colloquial) negation of $A$ are to be meaningful. Thus the sentence fohn gave up smoking presupposes that John previously smoked. Fack knows that there is a university in Regensburg presupposes that Regensburg does indeed have a university. The utterance $A s$ a doctor I realize how dangerous this symptom is presupposes that the speaker is a physician. These presuppositions are not part of the content of the sentences but rather preconditions to them being meaningful at all. Such presuppositions, being again matters of empirical fact, cannot be accounted for syntactically.

Invalid presuppositions also appear in the following special cases:

(4) Definite descriptions with unfulfilled normality conditions: Description terms as Russell's book or George VI's son have no meaning because the describing predicate fails to apply to exactly one object as the normality condition of descriptions requires. Whether this condition holds or not is again an empirical question, not a syntactic one.

(5) Empty generalizations: In ordinary discourse the sentence All of Fohn's children have red hair is meaningless if John does not have any children. In general a sentence of the form All A's are $B$ is only meaningful if there are $A$ s. Such a sentence thus presupposes that sentence As exist. This should not in every case be understood to mean that there must exist 'real objects' which are $A$ s-sentences like All the Greek Gods were assimilated into the Roman Pantheon indicate to the contrary that they can also be 'possible objects'. These presuppositions of descriptions and generalizations were first noticed by P. F. Strawson.

(6) Quantifying into intensional contexts: W. V. Quine (1953) has repeatedly emphasized that it is senseless to quantify into intensional contexts, as in the sentences There is a number $x$ such that $x$ is necessarily greater than 7 or There is a person $x$ such that Philip is unaware that $x$ denounced Catiline. A quantification of this sort is only meaningful under the normality condition that the use of the predicate depends solely upon the extension and not upon the intension of the argument indicated by the variable as is the case in deontic contexts like There is a person $x$ who is obliged to examine the students (see Kutschera (1973), section r.6).

In this paper we propose to discuss how the problem of grammatically well-formed but meaningless expressions can be handled within the 
general framework of intensional semantics developed by R. Montague. ${ }^{1}$ This semantic system refers primarily to an artificial language $L$ of the logic of types and will be treated exclusively as such in the following. Rules for the interpretation of a natural language $S$ can be derived from this system only when an analysing relation between the expressions of $L$ and those of $S$ is defined.

Let us first take a general look at possible courses toward solving our problem. We shall disregard solutions which syntactically exclude meaningless expressions as being not well-formed. One could in this manner, for instance with respect to (I), introduce a many-sorted language with several object domains and several varieties of constants and variables of the same category so that every single-place predicate would be defined for exactly one object domain. The examples given under ( $\mathrm{I}$ ), however, already indicate that this is a hopeless undertaking since the predicates of a natural language are not all defined on sets that can be delineated by such simple classifications as 'animal', 'human', 'abstract object', etc. Such an attempt becomes even more dubious in case (2) and collapses completely in cases (3)-(6). The problem permits only semantic solutions if unpleasant interference between syntax and semantics is to be avoided. ${ }^{2}$

Semantic solutions offer themselves in the following ways:

(a) Completing the semantic interpretation: We might stipulate, for instance, that a basic predicate takes on the value false for an argument for which it is not defined-I7 runs and The moon laughs are then false sentences, just as Odysseus is shaving and Eisenhower is sick. Furthermore the interpretation of description terms is extended, for instance in the sense of Frege, in such a way that they have a meaning even when the normality condition is not met. Generalizations are interpreted in such a way that they are true when their presuppositions are not fulfilled. ${ }^{3}$ And in the cases mentioned under (3), finally, one can resort to the device of including the presuppositions into the assertions. Thus the sentence fohn gave up smoking would be interpreted as meaning Fohn used to smoke but doesn't any more. Supplementing the semantic interpretation in this way has been the customary procedure in logic since Frege.

(b) Incomplete 2-valued interpretations: One uses a 2-valued semantics but permits interpretations which do not assign a meaning to every syntactically well-formed term. Functors can then be interpreted as partial

1 Reference will be mainly to Montague (1970).

2 Syntax and semantics interfere with one another, for instance, if forming a description term is permitted only when the normality condition is provable.

${ }^{3}$ For a complete interpretation of generalizations in intensional contexts, see 2.3.r(c). 
functions so that a sentence $F(a)$ remains meaningless when the reference of $a$ does not belong to the domain over which $F$ is defined. Expressions involving presuppositions are only interpreted if these are valid. This is the solution proposed by D. Scott (1970).

(c) 3-valued interpretations: Along with the truth values of sentences true and false one introduces a third value meaningless, and assigns meaningless proper names an object meaninglessness as reference and thus constructs a 3-valued semantics. A 3-valued semantics has been offered for predicate logic for instance by Woodruff ( 1970 ), but he only considers such meaningless expressions as arise from the use of meaningless proper names. For this reason we shall discuss a more general 3-valued semantics below.

(d) Sets of 2-valued interpretations: Proceeding from the idea that meaningless expressions arise when only limited information is available about the interpretation of a language, one represents such limited semantic information by the set $T$ of 2-valued interpretations $M$ which are eligible relative to that information. $T$ then assigns to an expression $A$ the value $\alpha$, if for all $M \in T, M(A)=\alpha$. If there is no such $\alpha$, then $T$ is not defined for $A$. This procedure leads therefore to considering a term meaningless if the semantic information is compatible with different interpretations for this term. If, for instance, a predicate $F(x)$ is only defined over a proper subset $U^{\prime}$ of the object domain $U$, then all possible continuations of this partial function on $U$ are considered as possible interpretations of $F$. If the constant $a$ designates an object from $U-U^{\prime}$, these interpretations provide different values for $F(a)$ so that $F(a)$ is characterized as meaningless with respect to the set of these interpretations. And if the normality condition for descriptions does not hold, then every assignment of an object to this term would be a possible interpretation of the term so that it again is meaningless relative to the set of these interpretations.

Such an approach has been developed especially by B. van Fraassen (1969). It refers, however, only to the language of elementary predicate logic in an extensional interpretation.

Completing semantic interpretations in accordance with proposal (a) leads to several inadequacies in the semantic analysis of natural language sentences. First of all one has to determine which predicates are to be basic predicates. For instance should sick be taken as a basic predicate and healthy as not sick or vice versa. Both cannot be taken as basic because otherwise the sentence Eisenhower is neither sick nor healthy would be correct, in contradiction to the analytic sentence Anyone who is not healthy is sick. Such conventions are, however, very artificial for natural languages and 
they always end up by making sentences false which ordinarily are considered true: If work and to be lazy are basic predicates, then the sentence Anyone who never works is lazy is false by virtue of the new semantic conventions, since numbers do not work. Most importantly, however, the distinction between the assertion of a sentence and its presupposition gets lost and its meaning is thereby distorted. If one interprets the sentence fohn gave up smoking to mean fohn used to smoke and doesn't any more then the negation of this sentence maintains fohn didn't use to smoke or fohn still smokes and in contradistinction to fohn didn't give up smoking is true even if John never smoked.

The proposal (a) therefore offers no satisfactory solution to our problem, so we can limit ourselves henceforth to a discussion of proposals (b), (c) and (d), i.e. those concerning partial interpretations which do not assign every term a meaning. Our primary objective will be to work out and compare these proposals within the framework of Montague's semantics. It will turn out that (b) and (c) have essentially the same effect while proposal (d) does not lead to satisfactory results.

We will start off in the next section by defining the ordinary, complete, 2-valued interpretations in the sense of Montague, in order to elucidate where the partial interpretations differ from them. ${ }^{1}$

\section{Fundamentals of intensional semantics}

\section{I. The syntax of $L$}

The language $L$ upon which intensional semantics is based is constructed in the following way:

We determine first the categories of $L$-expressions.

\section{I.I.}

(a) $\sigma, v$ are categories.

(b) If $\tau, \rho$ are categories, then $\tau(\rho)$ is also a category.

(c) If $\tau$ is a category, then $\iota(\tau)$ is also a category.

$\sigma$ is the category of sentences, $v$ the category of proper names, $\tau(\rho)$ is the category of functors which produce expressions of category $\tau$ from arguments of category $\rho, \iota(\tau)$ is the category of intensions of expressions of the category $\tau$.

1 These definitions are taken from Kutschera (1975) where they are intuitively explained. 
The alphabet of $L$ consists of the symbols $\lambda, \equiv, \mu, \delta,($,$) and infinitely$ many constants and variables of every category. The category of an expression will often be noted by use of an upper index.

The symbol * is not a part of $L . A\left[{ }^{*}\right]$ is a finite series of basic $L$-symbols together with this symbol, and $A[a]$ is the expression resulting from replacement in $A\left[{ }^{*}\right]$ of all occurrences of * by $a$.

The well-formed expressions or terms of $L$ are determined by

\section{I.2.}

(a) Constants of the category $\tau$ of $L$ are terms of the category $\tau$ of $L$.

(b) If $F$ is a term of the category $\tau(\rho)(\tau \neq \iota)$ and $a$ is a term of the category $\rho$ of $L$, then $F(a)$ is a term of the category $\tau$ of $L$.

(c) If $A[a]$ is a term of the category $\tau, a$ is a constant of the category $\rho$ and $x$ is a variable of the category $\rho$ of $L$ which does not occur in $A[a]$, then $\lambda x(A[x])$ is a term of the category $\tau(\rho)$ of $L$.

(d) If $a$ and $b$ are terms of the same category of $L$, then $a \equiv b$ is a term of the category $\sigma$ of $L$.

(e) If $A$ is a term of the category $\tau$ of $L$, then $\mu(A)$ is a term of the category $\iota(\tau)$ of $L$.

(f) If $A$ is a term of the category $\iota(\tau)$ of $L$, then $\delta(A)$ is a term of the category $\tau$ of $L$.

Where brackets are not necessary to delineate clearly the range of an operator $\lambda, \mu$ or $\delta$, they will be left out in the following.

$L_{1}$ shall be that sublanguage of $L$ in which the operators $\mu$ and $\delta$ do not appear and only constants and variables of those categories which can be constructed solely in accordance with rules (a) and (b) of 2.I.I.

\subsection{Extensions}

For clarity's sake the semantics of $L$ will be constructed in several stages, in the first of which the expressions of $L$ are assigned only extensions. For this purpose we will restrict ourselves to the sublanguage $L_{1}$.

2.2.I. Let $E_{\tau, U}$ be the set of possible extensions of the terms of $L_{1}$ of the category $\tau$ relative to the object domain $U$.

(a) $E_{v, U}=U$

(b) $E_{\sigma, U}=\{w, f\}$

(c) $E_{\tau(\rho), U}=E_{\tau, U},{ }^{E}, U$ 
$w$ represents the truth value true and $f$ the truth value false. $A^{B}$ is the set of functions with domain $B$ and a range included in $A$.

2.2.2. An extensional interpretation of $L_{1}$ over the (non-empty) object domain $U$ is a I-place function $M$ with the following properties:

(a) $M(a) \in E_{\tau, U}$ for all constants $a$ of the category $\tau$.

(b) $M(F(a))=M(F)(M(a))$ for all terms in accordance with 2.1.2(b).

(c) $M(\lambda x A[x])$ is that function $f$ from $E_{\tau(\rho), U}$ for which $f\left(M^{\prime}(b)\right)=$ $M^{\prime}(A[b])$ holds for all $M^{\prime}$ with $M^{\prime} \overline{\bar{b}} M$. The term $\lambda x A[x]$ is formed in accordance with 2.I.2(c) and the constant $b$ of the same category as $x$ shall not occur in $\lambda x A[x]$.

(d) $M(a \equiv b)=w$ iff $M(a)=M(b)$ for all terms in accordance with 2.1.2(d).

In (c) $M^{\prime} \overline{\bar{b}} M$ means that the two interpretations $M$ and $M^{\prime}$ differ at most in the values they assign the constant $b$.

2.2.3. We define following Montague:

(a) $\wedge x^{\tau} A:=\lambda x^{\tau} A \equiv \lambda x^{\tau}\left(x^{\tau} \equiv x^{\tau}\right)$

(b) $\neg A:=A \equiv \wedge x^{\sigma}\left(x^{\sigma}\right)$.

(c) $A \wedge B:=\wedge x^{\sigma(\sigma)}\left(B \equiv\left(x^{\sigma(\sigma)}(A) \equiv x^{\sigma(\sigma)}(B)\right)\right)$

(d) $A \vee B:=\neg(\neg A \wedge \neg B)$

(e) $A \supset B:=\neg A \vee B$

(f) $\vee x^{\tau} A:=\neg \wedge x^{\tau} \neg A$.

2.2.4. It is often useful to introduce names for non-existing objects like Odysseus. Taking $U$ as the set of 'possible objects' and a subset $U^{\prime}$ of $U$ as the set of 'real objects', then where $E$ is a constant of category $\sigma(v)$ and $a$ is a constant of category $v$ we postulate

(e) $M(E(a))=w$ iff $M(a) \in U^{\prime}$.

If we then define

(g) $\wedge \cdot x^{\nu} A\left[x^{\nu}\right]:=\wedge x^{\nu}\left(E\left(x^{\nu}\right) \supset A\left[x^{\nu}\right]\right)$

(h) $\vee \cdot x^{\nu} A\left[x^{\nu}\right]:=\neg \wedge \cdot x^{v} \neg A\left[x^{v}\right]$

the following principles hold

$$
\begin{aligned}
& A[a] \wedge E(a) \supset \vee . x A[x] \\
& \wedge . x A[x] \wedge E(a) \supset A[a] .
\end{aligned}
$$


This means that quantification with $\Lambda$. and $\vee$. takes into account only existing objects.

2.2.5. Descriptions can be introduced in $L_{1}$ in such a way that $\iota x A[x]$ is a term of $L_{1}$ of category $\tau$ if $A[b]$ is a term of category $\sigma, b$ a constant and $x$ a variable of category $\tau$ of $L_{1} ; x$ should not occur in $A[b]$. It can then be postulated in extension of $\mathbf{2 . 2 . 2}$ :

(f) $M(x A[x])=\alpha$

if there is exactly one $M^{\prime}$ such that $M^{\prime} \underset{a}{=} M$ and $M^{\prime}(A[a])=w$ and if for this $M^{\prime} M^{\prime}(a)=\alpha(a$ being a constant of the same category as $x$ and one which does not occur in $\iota x A[x]$; otherwise we let $M(\iota x A[x])=M\left(a_{o}\right)$ where $a_{o}$ is a fixed constant of the category $\tau$.

If the description operator is only to refer to existing objects, it can be defined by

(i) . . $x^{\nu} A\left[x^{v}\right]:=\iota x^{v}\left(A\left[x^{\nu}\right] \wedge E\left(x^{v}\right)\right)$.

\subsection{Intensions}

Considering now the assignment of intensions to the expressions of $L$ let us shift over from $L_{1}$ to $L . L$ can be characterized as a modal language of the logic of types. According to Carnap the intension of an expression should be taken as that function which determines its extension for every possible world. $i \in I$ are to be indices for these possible worlds.

The extension of a functional expression such as it is necessary that $p$ is often dependent not only on the extension but also on the intension of the argument $p$. Thus the arguments of the functor it is necessary that ... are assigned the category $\iota(\sigma)$ for propositions (intensions of sentences) instead of the category $\sigma$ for sentences. Since however the intension of $p$ can be expressed by $\mu(p)$, it is possible to maintain, as Frege did, that the extensions of functional expressions always depend on the extensions of their arguments if one writes it is necessary $(\mu(p))$ instead of it is necessary that $p$.

Supplementing the conventions 2.2.I by

(d) $E_{\iota(\tau), U}=E_{\tau, U}^{I}$

we define:

2.3.I. An intensional interpretation of $L$ over the (non-empty) universe 
domain $I$ with the (non-empty) object domain $U$ is a 2-place function $M_{i}(A)$ such that the following holds for all $i \in I$ :

(a) $M_{i}(a) \in E_{\tau, U}$ for all constants $a$ of the category $\tau$.

(b) $M_{i}(F(a))=M_{i}(F)\left(M_{i}(a)\right)$ for all terms according to 2.1.2(b).

(c) $M_{i}(\lambda x A[x])$ is that function $f$ from $E_{\tau(\rho), U}$, for which: $f\left(M_{i}^{\prime}(b)\right)=$ $M_{i}^{\prime}(A[b])$ holds for all $M^{\prime}$ with $M^{\prime}=M$, and $M_{j}^{\prime}(b)=M_{i}(b)$ for all $j \neq i$ from $I$. The term $\lambda x A[x]$ is constructed in accordance with 2.r.2(c) and the constant $b$ of the same category as $x$ shall not occur in $\lambda x A[x]$.

(d) $M_{i}(a \equiv b)=w$ iff $M(a)=M(b)$ for all terms according to 2.I.2(d).

(e) $M_{i}(\mu(A))=\lambda^{*} i M_{i}(A)$.

(f) $M_{i}(\delta(A))=M_{i}(A)(i)$.

$\lambda^{*}$ is to be a symbol of the metalanguage for functional abstraction. $M^{\prime} \overline{\bar{b}} M$ now means that the intensional interpretations $M$ and $M^{\prime}$ differ at the most with regard to the values $M_{i}(b)$ and $M_{i}^{\prime}(b)$ for any number of $i \in I$.

$M_{i}(A)$ is the extension of the term $A$ in world $i, \lambda^{*} i M_{i}(A)$ its intension.

If the variable $x$ in $\lambda x A[x]$ does not occur within the scope of an operator $\mu$, then one can also define: $M_{i}(\lambda x A[x])$ is that function $f$ from $E_{\tau}[\rho]_{, v}$ for which $f\left(M_{i}^{\prime}(b)\right)=M_{i}^{\prime}(A[b])$ holds for all $M^{\prime}$ with $M^{\prime}{ }_{\bar{b}} M$; for then for all $M^{\prime}$ and $M^{\prime \prime}$ with $M^{\prime}=M, M_{b}^{\prime \prime} \underset{b}{=} M$ and $M_{i}^{\prime}(b)=M_{i}^{\prime \prime}(b)$ we have $M_{i}^{\prime}(A[b])=M_{i}^{\prime \prime}(A[b])$. This does not hold however when $\lambda x A[x]$ is the expression $\lambda x^{v} G^{\sigma\left(\gamma_{1}(\sigma)\right)}\left(\mu\left(F^{\sigma(v)}\left(x^{v}\right)\right)\right)$ for which $\mu(F(a))$ can depend on the intension of $a$, i.e. on the values of $M_{j}(a)$ with $j \neq i$. In this case the expression $\lambda x G(\mu(F(x)))$, which is to be interpreted as a function from $E_{\sigma(v), U}$, has no reasonable meaning. The construction of the terms $\lambda x A[x]$, however, cannot be restricted to those cases in which $x$ does not occur in the scope of an operator $\mu$ since there are also interpretations of $G$ and $F$ for which the truth value of $G(\mu(F(a)))$ does not depend on the intension but only upon the extension of $a$. Such contexts are, for instance, deontic contexts like $O(\mu(F(a)))$ (i.e. $F(a)$ is obligatory). In such contexts we cannot do without terms such as $\lambda x A[x], \wedge x A[x]$, or $\mathrm{V} x A[x]$. It is therefore necessary to permit syntactically the construction of all the terms $\lambda x A[x]$ and they must then be interpreted in such a way that they have the usual meaning if the normality condition obtains, i.e. if $M_{i}(A[a])$ does not depend upon the $M_{j}(a)$ with $j \neq i$. And that is what condition (c) does. 
In $L$ we can define, besides the operators under 2.2.3, modal operators as

(j) $\square A:=\mu(A) \equiv \mu\left(\wedge x^{\nu}\left(x^{\nu} \equiv x^{\nu}\right)\right)$

(k) $\diamond A:=\neg \square \neg A$.

If for every $i$ a set $U_{i}$ of objects existing in $i$ is given with $U_{i} \subset U$, then operators for quantifying over existing objects can be introduced in analogy to 2.2.4 with the help of the existence predicate $E$ for which $M_{i}(E(a))=w$ now holds iff $M_{i}(a) \in U_{i}$. Since statements about the quantity of objects existing in a world are to be formulated with such quantifiers, no sentence of the form $I t$ is necessary that there are exactly $k$ objects is analytically true unless all the $U_{i}$ have the same cardinality.

\subsection{Pragmatic relations}

We shall disregard here the meaning dependencies which derive from the linguistic context in which an expression occurs (Kutschera (1975)), and take account only of the fact that the meaning of an utterance may depend on its non-linguistic, situational context, e.g. by making use of indexical expressions. If the semantically relevant pragmatic parameters are summarized in an index $j \in \mathcal{F}$ - the point of reference of an utterance - then we can define:

2.4.I. A pragmatic interpretation of $L$ over the universe with domain $I$ and the (non-empty) index set $\mathcal{F}$ is a 3 -place function $M_{i, j}$ such that for all $j \in \mathcal{F}, M_{i, j}$ is an intensional interpretation of $L$ over $I$ in the sense of 2.3.I. We call $M_{i, j}(A)$ the extension of statement $A$ at the point of reference $j$ with regard to $i, \lambda^{*} i M_{i, j}$ its intension, $\lambda^{*} j M_{i, j}(A)$ the extension of $A$ with regard to $i$ and $\lambda^{*} i j M_{i, j}(A)$ the intension of $A$.

\section{Partial 2-valued interpretations}

\section{I. Extensions}

After these preparatory steps we can now take up method (b) of section $\mathbf{I}$ and specify in greater detail what is meant by an incomplete 2-valued interpretation of the language $L$. We begin again by assigning extensions to the expressions of $L_{1}$.

Every complete 2-valued interpretation of $M$ over $U$ in the sense of 2.2.2 assigns a possible extension from $E_{\tau(\rho), U}$ to a functional constant $F$ of the category $\tau(\rho)$. These functions are defined for all arguments from 
$E_{\rho, U}$. If $M(a) \in E_{\rho, U}$ has been defined, then $M(F(a))$ is also defined. Thus in handling case (I) from section I of incompletely defined functors by the use of incomplete 2-valued interpretations, partial functions must be permitted as possible extensions of functors. The set of possible extensions must therefore be defined in the following way:

3.I.I.

$$
\begin{aligned}
& E_{v}^{+}, U=E_{v, U} \\
& E_{\sigma, U}^{+}=E_{\sigma, U} \\
& E_{\tau(\rho), U}^{+}=E_{\tau, U}^{+}\left(E_{\rho}^{+}, U\right) .
\end{aligned}
$$

$A^{(B)}$ is to be the set of partial functions from $B$ into $A$, i.e. functions with a domain included in $B$ and a range included in $A .1$

If $E_{\tau, U}$ in 2.2.2 is everywhere replaced by $E_{\tau}^{+}$, if no demand is made in (a) that $M(a)$ be defined for all constants $a$ of the categories $v$ and $\sigma$, and if the conditions $(b)-(d)$ are taken to hold only in the case where the values of $M$ in the recursive conditions are defined, then we get the definition of the concept of a partial extensional interpretation of $L . M^{\prime}=M$ in condition $(c)$ is now to indicate that $M^{\prime}(b)$ or $M(b)$ can also be undefined. Therefore we attach to $(c)$ the supplementary condition that $M^{\prime}(b)$ should be defined. The conditions $f\left(M^{\prime}(b)\right)=M^{\prime}(A[b])$ in $(c)$ is meant to include that $f\left(M^{\prime}(b)\right)$ be undefined iff $M^{\prime}(A[b])$ is undefined.

3.I.2. If definition (a) from 2.2.3 is taken over, then $M(\wedge x A[x])=w$ holds in case $M(\lambda x A[x])$ is a complete function taking on the value $w$ for every argument. Otherwise $M(\wedge x A[x])=f$ holds since $M(\lambda x A[x])$ is always defined. Consequently according to definition (f) from 2.2.3 $\mathrm{V} x A[x]$ is true in every case where $M(\lambda x A[x])$ is a partial function, no matter whether this function takes on the value $w$ for a particular argument or not. Thus definition (a) is not suitable. In order to arrive at quantifiers $\wedge x$ and $\vee_{x}$ referring only to the objects for which $A[x]$ is defined we introduce an operator $\sim$ such that $a \sim b$ is a term of $L_{1}$ if $a$ and $b$ are terms of the same category $\tau(\rho)$, and postulate:

$M(a \sim b)=w$ iff $M(a)$ and $M(b)$, restricted to the common domain of definition, are identical - in case this domain is not empty.

1 If $M\left(F^{\tau(\rho)}\right)$ is the totally undefined function from $E^{+}{ }_{\tau}(\rho), v$, then $F^{\tau(\rho)}$ remains undefined. Since we also wish to admit the case where $M$ is undefined for some constants - thour: $h$ it is
of no special interest $n$ the present context - we can also include totally undefined fun:tions into the set $E_{{ }^{+}(\rho), v}$. 
With

(a') $\wedge x^{\tau} A\left[x^{\tau}\right]:=\lambda x^{\tau} A\left[x^{\tau}\right] \sim \lambda x^{\tau}\left(x^{\tau} \equiv x^{\tau}\right)$

and (f) we can then introduce suitable quantifiers and keep the rest of the definitions in 2.2.3.

Definition $\left(a^{\prime}\right)$, however, does not solve the problem of colloquial generalizations of the form All As are B mentioned in section r. In common usage such a sentence is only about the $A$ s - i.e. it has a truth value iff there are $A$ s and $B$ is defined for all $A$ s. For representing such sentences restricted generalizations can be introduced, for instance by stipulating

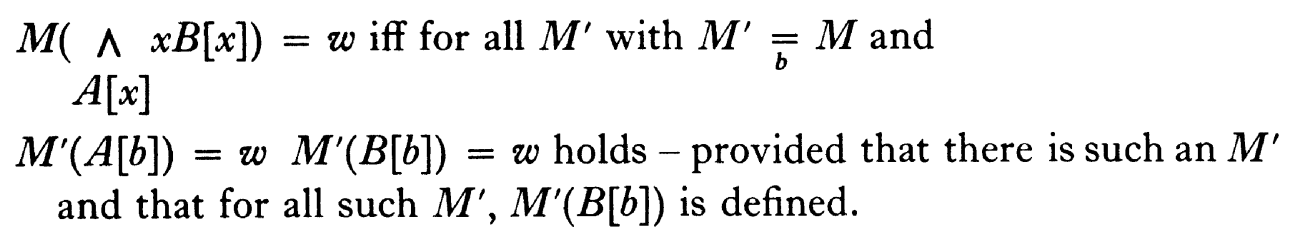

Names of non-existing objects can be handled as in 2.2.4. However, instead of defining descriptions as in 2.2.5 as well for the case where the normality condition is not met, using partial interpretations we can restrict the definition to the normal case, i.e. the case in 2.2.5(i).

\subsection{Intensions}

If in extension of 3.r.I.we postulate

$$
E_{\imath(\tau), U}^{+}=E_{\tau, U}^{(I)}
$$

we can define partial intensional interpretations in the same correspondence to 2.3.I as we defined extensional interpretations in correspondence to 2.2.2. Having, however, emphasized in sections $I$ and 2.3 that quantification into intensional contexts is only meaningful under the normality condition, 2.3.I(c) must be replaced by:

(c') $M_{i}(\lambda x A[x])$ is that function $f$ from $E_{\tau}^{+}(\rho), U$ for which $f\left(M_{i}^{\prime}(b)\right)=$ $M_{i}^{\prime}(A[b])$ holds for all $M^{\prime}$ with $M^{\prime} \overline{\bar{b}} M$ and $M_{i}^{\prime}(b)$ defined - provided that for all $M^{\prime}, M^{\prime \prime}$ with $M^{\prime} \overline{\bar{b}} M, M^{\prime \prime} \overline{\bar{b}} M$ and $M_{i}^{\prime}(b)=$ $M_{i}^{\prime \prime}(b)$ (for which both these values are defined) $M_{i}^{\prime}(A[b])=$ $M_{i}^{\prime \prime}(A[b])$. Otherwise $M(\lambda x A[x])$ shall be the totally undefined function from $E_{\tau(\rho), U}^{+} \lambda x A[x]$ shall be a term according to 2.I.2(c) in which the constant $b$ of the same category as $x$ does not occur. 
With regard to partial pragmatic interpretations we also permit that $M_{i, j}(A)$ be not defined for all $j \in \mathcal{F}$.

The introduction of intensions and pragmatic relations provides a suitable framework for treating presuppositions.

3.2.I. A sentence $B$ of $L$ is a presupposition of $A$ relative to interpretation $M$ iff for all $i \in I$ and $j \in \mathcal{F} M_{i, j}(A)$ is only defined if $M_{i, j}(B)=w$. If $M_{i, j}(B)$ is not dependent on $j$, then we call $B$ a purely intensional presupposition, and if $M_{i, j}(B)$ is not dependent upon $i$, we call $B$ a purely pragmatic presupposition.

According to this definition a sentence has an intension (as partial function) in a world $i$ even if it has no extension in $i$ because of a nonfulfilled presupposition. A description whose normality condition is not met is thus not completely without meaning, it just does not denote anything.

It is obvious that material implications cannot be used for the definition of presuppositions, if $B$ is called a presupposition of $A$ iff $A$ and non- $A$ both imply $B$. Otherwise every factually correct sentence $B$ would be a presupposition to every other sentence $A$. Just as obvious, however, is the fact, that this implication cannot be understood in the sense of an entailment with reference to a set of interpretations (such that every interpretation which would make $A$ true or false would make $B$ true) because the question of whether $B$ is a presupposition of $A$ depends on the interpretation of $A$ and $B$ as long as we are considering the general case under point (3) in section I and not only the special cases in (4) and (5). For this reason the proposal of van Fraassen (1969) for handling presuppositions is not generally applicable. Only by reference to possible worlds and intensions can a general dependency of the assignment of an extension to $A$ upon the truth value of $B$ be determined with respect to one and the same interpretation $M$.

\section{3-valued interpretations}

We turn now to the proposal to account for meaningless but grammatically well-formed expressions within the framework of a 3 -valued logic. Since there is a multiplicity of 3 -valued logics, we must first consider the principles such a logic should be based upon.

We wish to interpret the third value of sentences along with $w$ and $f-$ we will call it $o^{\sigma}$ - as 'meaningless' in the sense of 'left indeterminate in truth value by the semantic conventions'. We adopt an extensive inter- 
pretation of the value $o^{\sigma}$ according to which a sentence is characterized as meaningless if one of its components is meaningless. The orientation is towards partial 2-valued interpretations which also assign no truth value to a sentence if they are not defined for its components. The object is generally to establish a close correspondence between 2- and 3 -valued interpretations so that for every 3-valued interpretation $N$ there is a 2-valued interpretation $M$ which assigns all sentences $A$ with $N(A) \neq o^{\sigma}$ the same truth value as $N$, and vice versa: i.e. for every 2-valued interpretation $M$ there is a 3-valued interpretation $N$ which assigns every sentence $A$ for which $M(A)$ is defined the same truth value as $M$. On the basis of this correspondence the theorems of 3 -valued ogic will be exactly those of 2 -valued logic. In natural languages we use in fact 2-valued logic, excepting, however, the metatheoretical principle of bivalence which requires that every sentence be true or false. 1

A 3 -valued logic can now be constructed on these lines corresponding either to the semantics of section 2 or to the semantics of section 3 . We will only carry out the latter project here since the use of partial functions is unavoidable, as for instance in the treatment of presuppositions of indirect sentences. The truth value of a sentence of the form $F(\mu(A))-$ e.g. Fohn said that fack gave up smoking - depends on the partial function $\lambda^{*} i M_{i}(A)$ and is not invariant with regard to a change in its domain of definition. And in general the reference to the frequent occurrence of only partially defined concepts which we made in section I already suggests strongly the use of partial functions as possible extensions.

\section{I. Extensions}

We will define first extensional 3 -valued interpretations for the language $L_{1}$ and assign every category $\tau$ a single object 'meaninglessness' $o^{\tau} . o^{\sigma}$ will be assigned to a meaningless sentence, $o^{v}$ to a meaningless proper name. The objects $o^{\tau(\rho)}$ will be introduced below.

The set of possible extensions of the category $\tau$ for the object domain is determined in analogy to 2.2.1 in the following way:

\section{I.I.}

$$
\begin{aligned}
& E_{v, U}^{*}=U \cup\left\{o^{\nu}\right\} \\
& E_{\sigma, U}^{*}=\left\{w, f, o^{\sigma}\right\}
\end{aligned}
$$

${ }^{1}$ For the distinction between accepting the object language principle of tertium non datur and accepting the metalinguistic principle of bivalence see van Fraassen (1969). 
$E_{\tau(\rho), U}^{*}$ is the set of functions $f$ from $E_{\tau, U}^{*} E_{\rho}^{*}, U$ which take the value $o^{\tau}$ for the argument $o^{\rho}$.

$o^{\tau(\rho)}$ shall be that function from $E_{\tau(\rho), U}^{*}$ which takes the value $o^{\tau}$ for all arguments.

4.I.2. An extensional 3-valued interpretation of $L_{1}$ over the (non-empty) object domain $U$ is a I-place function $N$ with the following properties:

(a) $N(a) \in E_{\tau, U}^{*}$ for all constants $a$ of $L_{1}$ of the category $\tau$.

(b) $N(F(a))=N(F)(N(a))$ for all terms in accordance with 2.1.2(b).

(c) $N(\lambda x A[x])$ is that function $f$ from $E_{\tau(\rho), U}^{*}$ with $f\left(N^{\prime}(b)\right)=N^{\prime}(A[b])$ for all $N^{\prime} \bar{b}=N$ and $N^{\prime}(b) \neq o^{\rho} . \lambda x A[x]$ is a term according to 2.I.2(c) and the constant $b$ of the same category as $x$ shall not occur in $\lambda x A[x]$.

(d) $N\left(a^{\tau} \equiv b^{\tau}\right)=w$ for $N\left(a^{\tau}\right) \neq o^{\tau}$ and $N\left(a^{\tau}\right)=N\left(b^{\tau}\right) ; N\left(a^{\tau} \equiv b^{\tau}\right)=f$ for $N\left(a^{\tau}\right) \neq o^{\tau} \neq N\left(b^{\tau}\right)$ and $N\left(a^{\tau}\right) \neq N\left(b^{\tau}\right)$; and otherwise $N\left(a^{\tau} \equiv\right.$ $\left.b^{\tau}\right)=o^{\sigma}-$ for all terms according to 2.I.2(d).

The intuitive ideas for the construction of a 3 -valued logic as formulated above can then be made precise in the following way:

4.I.3. An extensional 3-valued interpretation $N$ and an extensional partial interpretation $M$ (in the sense of 3.1 ) shall be called correlated if

(a) $M$ and $N$ are based on the same object domain;

(b) for all constants $a^{\tau}$ with $\tau=v, \sigma M\left(a^{\tau}\right)$ is defined iff $N\left(a^{\tau}\right) \neq o^{\tau}$; if $M\left(a^{\tau}\right)$ is defined, then $M\left(a^{\tau}\right)=N\left(a^{\tau}\right)$;

(c) for all constants $a^{\tau(\rho)}, M\left(a^{\tau(\rho)}\right)$ is the totally undefined function from $E_{\tau(\rho), U}^{+}$iff $N\left(a^{\tau(\rho)}\right)=o^{\tau(\rho)}$; otherwise $M\left(a^{\tau(\rho)}\right)=N\left(a^{\tau(\rho)}\right) / D\left(N\left(a^{\tau(\rho)}\right)\right.$.

$D(N(a))$ is the set of arguments $\neq o^{\rho}$, not assigned the value $o^{\tau}$ by $N(a)$, while $f / E$ is the function $f$ restricted to $E$.

This correlation is a one-to-one correspondence between all 3 -valued and all partial interpretations.

4.I.4. If $N$ and $M$ are correlated then everything which applies to the constants of $L_{1}$ according to 4 . I.3 applies also to all terms of $L_{1}$.

This statement can be proved by induction on the degree of the terms, i.e. on the number of occurrences of logical operators in them, where brackets which express function-argument positioning according to 2.1.2(b) are also counted as operators. 
We define:

4.I.5. A partial interpretation $M$ satisfies a sentence $A$ weakly if $M(A)=w$ or if $M(A)$ is undefined. A 3 -valued interpretation $N$ satisfies $A$ weakly if $N(A) \neq f .{ }^{1} A$ shall be called weakly valid if all partial interpretations satisfy $A$ weakly. Furthermore $A$ shall be called weakly 3-valid if all $3^{-}$ valued interpretations satisfy $A$ weakly.

From 4.I.4 we then obtain the theorem:

4.I.6. The weakly 3 -valid sentences of $L_{1}$ are exactly the weakly valid sentences of $L_{l}$.

If the 3-valued semantics had been constructed corresponding to the semantics of section 2 instead of section 3 , we would have obtained in place of 4.2.6 the theorem: The weakly 3 -valid sentences are exactly the logically true sentences, i.e. the sentences satisfied by all complete 2-valued interpretations.

For the definitions of 2.2.3 corresponding remarks apply as were made in 3.I.2. We postulate

$$
\begin{aligned}
& N\left(a^{\tau} \sim b^{\tau}\right)=w, \text { if } N\left(a^{\tau}\right) \neq o^{\tau} \neq N\left(b^{\tau}\right) \text { and } N\left(a^{\tau}\right) / D\left(N\left(a^{\tau}\right)\right) \cap D\left(N\left(b^{\tau}\right)\right) \\
& =N\left(b^{\tau}\right) / D\left(N\left(a^{\tau}\right)\right) \cap D\left(N\left(b^{\tau}\right)\right) ; N\left(a^{\tau} \sim b^{\tau}\right)=o^{\tau}, \text { if } N\left(a^{\tau}\right)=o^{\tau} \text { or } N\left(b^{\tau}\right)= \\
& o^{\tau} ; \text { and otherwise } N\left(a^{\tau} \sim b^{\tau}\right)=f .
\end{aligned}
$$

Names of non-existing objects and description terms can be treated in direct analogy to 3.I.

\subsection{Intensions}

The definition of 3 -valued intensional interpretations also follows directly from 2.3 and 3.2. We let

$$
E_{\imath(\tau), U}^{*}=E_{\tau, U}^{I} \text {. }
$$

$o_{\iota(\tau)}^{*}$ is to be that function from $E_{\imath}^{*(\tau)}, U$ which assigns every $i \in I$ the value $o^{\tau}$. In correspondence to 3.2 condition (c) of 2.3.I in the definition of intensional 3 -valued interpretation takes the form:

$\left(\mathrm{c}^{\prime \prime}\right) N_{i}(\lambda x A[x])$ is that function $f$ from $E_{\tau(\rho), U}^{*}$ for which $f\left(N^{\prime}(b)\right)=$ $N^{\prime}(A[b])$ holds for all $N^{\prime}$ with $N^{\prime} \overline{\bar{b}} N$ and $N^{\prime}(b) \neq o^{\rho}$ - in case $N_{i}^{\prime}(A[b])=N_{i,}^{\prime \prime}(A[b])$ holds for all $N^{\prime}, N^{\prime \prime}$ with $N^{\prime} \overline{\bar{b}} N, N^{\prime \prime} \overline{\bar{b}} N$,

1 The concept 'satisfies weakly' was introduced by Woodruff (1970). 
and $N_{i}^{\prime}(b)=N_{i}^{\prime \prime}(b) \neq o^{\rho}$. Otherwise $N_{i}(\lambda x A[x])=o^{\tau(\rho)}$. We choose $b$ as in 2.3.I.

The definitions and theorems 4.I.3 to 4.I.6 carry over to intensional 3 -valued interpretations, and introducing pragmatic interpretations in 3 -valued semantics requires no additional considerations either.

Summing up we can say: The 3-valued semantics we have sketched above derives in a simple and straightforward way from the semantics of partial 2-valued interpretations of section 3 by assigning undefined expressions the object 'meaninglessness'. 3-valued logics can, of course, also be constructed in quite different ways, but these are barred to us here since our intention has been to interpret the value 'meaninglessness' as 'indeterminate in value by a 2-valued semantic interpretation' and to let 3 -valued logic coincide with the 2-valued logic of natural languages.

\section{Sets of interpretations}

In building up our 3 -valued semantics we understood the characterization of terms as 'meaningless' in an extensional sense in which a sentence $A \vee B$, for instance, is meaningless if $A$ or $B$ is meaningless. In a narrower sense we could also call a sentence 'meaningless' if it could be assigned the value 'true' as well as 'false' by additional semantic stipulations. In this sense a sentence $A \vee B$ is not meaningless if $B$ is true even if $A$ is meaningless. And a sentence of the form $A \vee \neg A$ is never meaningless.

If meaningless expressions are to be understood in this way, (nonempty) sets $T$ of 2-valued interpretations $M$ suggest themselves as an adequate tool of semantic analysis. If $a$ is a term of $L$, it is interpreted by a set $T$ in such a way that

(a) $T(a)=\alpha$ in case $M(a)=\alpha$ holds for all $M \in T$, and

(b) $T(a)$ remains undefined, if there is no such $\alpha$.

Intuitively this procedure can be described thus: if only limited information about a (2-valued) interpretation of $L$ is available, take the set $T$ of all interpretations $M$ compatible with this information, and assign a term $a$ a meaning $\alpha$ if and only if this meaning can be derived from the given information, i.e. if and only if all $M \in T$ assign $a$ the value $\alpha$.

The sentences true for all such sets $T$ are obviously exactly the logically true sentences, i.e. the sentences true under all interpretations. Thus we get in a trivial way a result corresponding to 4.1.6.

We are, however, not concerned here with all the interpretations of $L$ 
that can be defined by arbitrary sets $T$. We have addressed ourselves rather to the problem, that an interpretation can be fixed for all constants of $L$ and can still be indeterminate for some terms of $L$. We are therefore interested primarily in those sets $T$ for which $T(a)$ is defined for all (or at least most) constants $a$, and must ask, therefore, if partial 2-valued interpretations can be represented by sets of complete 2 -valued interpretations, i.e. if working with sets of interpretations we can get the same results as working with partial interpretations.

Let us confine our attention to the assignment of extensions. If we take sets of complete interpretations in the sense of 2.2.2 we can account for the fact, that a term $a^{\tau(\rho)}$ denotes a partial function $f$ from $E_{\rho, U}$ into $E_{\tau, U}$ - we can set $T\left(a^{\tau(\rho)}\right)=f$, if $E \subset E_{\rho, U}$ is the most comprehensive set, such that $M\left(a^{\tau(\rho)}\right) / E=f$ holds for all $M \in T$-but those partial functions cannot appear as arguments of other functions since we are using $E_{\tau, U}$ instead of $E_{\tau, U}^{+}$as sets of possible extensions. We have seen, however, that the use of partial functions as arguments is indispensable for an adequate treatment of meaningless terms. We cannot, therefore, represent partial interpretations by sets of complete interpretations; so the use of such sets leads to unsatisfactory results.

There remains then only the recourse of using some sort of completed partial interpretations. Their definition is to be derived from that of a partial interpretation $M$ with the additional stipulation that if $M\left(a^{\tau}\right)$ is undefined, $M^{\prime}\left(a^{\tau}\right)$ is to be an arbitrary object from $E_{\tau, U}^{+}$for the completed interpretation $M^{\prime}$. If $T_{M}$ is the set of all completions of $M$, then $T_{M}$ is defined exactly for those terms $a$, for which $M$ is defined, and for them we have $M(a)=T_{M}(a)$.

But even if partial interpretations can be represented by sets of interpretations by this procedure, it is still quite unacceptable since the notion of a completed partial interpretation is intuitively wholly unreasonable. If $M^{\prime}$ is such an interpretation, $M^{\prime}\left(F^{\tau(\rho)}\right)$ a partial function, and $M^{\prime}\left(a^{\rho}\right)$ an object not belonging to the domain of this function, $M^{\prime}\left(F^{\tau(\rho)}\left(a^{\rho}\right)\right)$ is still supposed to be defined. Such a stipulation can, of course, not yield an intuitively acceptable concept of interpretation.

Partial interpretations cannot therefore be represented in a reasonable way by sets of interpretations.

The definition of an interpretation by a set of interpretations furthermore is not recursive: $T(A \supset B)$ does not depend directly on the values $T(A)$ and $T(B)$ and can be defined even if both $T(A)$ and $T(B)$ are undefined. This is not in accordance with the general semantic principle that the meaning of a sentence derives from the meaning of its constituents. 


\section{4}

And finally we want to quantify with the operator $\mu$ over interpretations $M_{i}$ in intensional semantics. If every $M_{i}$ is a complete interpretation, we cannot account for the presuppositions of $A$ by $\mu(A)$ and cannot use partial functions $\lambda^{*} i M_{i}(A)$ as arguments of functors. If, on the other hand, we were to work with sets $T_{i}$ of interpretations and quantify with $\mu$ over such sets, we would use values of interpretations as well as sets of interpretations in the recursive definition in a rather obscure fashion.

For these reasons the attempt to represent incomplete interpretations by sets of interpretations seems to be unsuccessful, or at least to become so complicated and artificial as to be without interest.

For a simple and adequate treatment of well-formed but meaningless terms there remain then only the 2-valued semantics of partial interpretations and 3 -valued semantics. Both come to the same thing on the definitions in section 4 . Since, however, in our 3 -valued interpretations the value 'meaningless' is understood in the sense of 'indeterminate under 2-valued semantic conventions', the notion of partial interpretation is to be regarded as the more fundamental one.

\section{REFERENCES}

Fraassen, B. C. van (1969). 'Presuppositions, supervaluations, and free logic'. In K. Lambert, ed., The Logical Way of Doing Things, pp. 67-91. New Haven Yale University Press.

Kutschera, F. v. (1973a). Einführung in die Logik der Werte, Normen und Entscheidungen. Freiburg.

- (1975). 'Grundzüge der logischen Grammatik'. To appear in S. J. Schmidt, ed. Pragmatic II. Munich.

Lambert, K. (ed.) (1970). Philosophical Problems in Logic. Dordrecht: Reidel.

Montague, R. (1970). 'Universal grammar', Theoria, 36, 373-98.

Quine, W. V. (1953). 'Reference and modality'. In W. V. Quine, From a Logical Point of View, pp. 139-59. Cambridge, Mass.

Scott, D. (1970). 'Advice on modal logic'. In Lambert (1970), pp. 143-73.

Woodruff, P. W. (1970). 'Logic and truth value gaps'. In Lambert (1970), pp. I2I-42. 